\title{
Correspondence Endogenic and latrogenic Diabetes Mellitus in Drug-naïve Schizophrenia: The Role of Olanzapine and its Place in the Psychopharmacological Treatment Algorithm
}

\author{
Dan Cohen*, ${ }^{1,2}$ and Marc De Hert ${ }^{3}$ \\ 'Department Chronic Psychiatry, Mental Health Care North-Holland North, Heerhugowaard, The Netherlands; '2Department of Epidemiology, \\ University Medical Center Groningen, Groningen, The Netherlands; ${ }^{3}$ University Psychiatric Centre, Catholic University Leuven, Kortenberg, Belgium
}

Neuropsychopharmacology (201 I) 36, 2368-2369; doi:I0.1038/npp.2011.94

The complex association of diabetes mellitus in patients with schizophrenia requires a twofold approach, that addresses both the illness-related and the iatrogenic side of the problem (Cohen 2006; De Hert et al, 2011).

In their study of the development of drug-induced diabetes mellitus in a cohort of 7139 young (mean age 29.4 years) antipsychotic-naïve schizophrenia patients, Nielsen et al (2010) not only contribute to our knowledge of the iatrogenic (= drug-induced) side of the problem, but also they - seemingly unknowingly - substantially improve our understanding of the illness-related side of this problem, although this contribution seems to have escaped the attention of the authors.

The authors report the presence of 91 cases of diabetes mellitus in drug-naïve patients with schizophrenia. Because of this, these patients were excluded from the study of drug-induced diabetes mellitus, as the outcome was already present before exposure to antipsychotic drugs. Not only can we calculate the size of the original drugnaïve study population $(N=7230$ (7139 included +91 excluded), but also the percentage, $1.26 \%$ (91/7230), of diabetes, which represents the prevalence of endogenic diabetes in a large population of young patients with schizophrenia.

How are we to read these results? First, a diagnostic procedure, specifically designed for a full diagnosis of all possibly undetected cases of diabetes was lacking; quite on the contrary, the diagnosis of diabetes in the study population was a clinical diagnosis, depending on both the patients - to present their symptoms to the GP - as on the GPs diagnostic acumen, quite comparable to the procedure in the general population. During the study period, the prevalence of diabetes in the general Danish population increased from $0.77 \%$ in 1997 to $0.99 \%$ in 2005 (Carstensen et al, 2008).

Three conclusions can be drawn. First, the authors have documented the minimal prevalence of endogenic diabetes

\footnotetext{
*Correspondence: Dr D Cohen, Department Chronic Psychiatry, Mental Health Care North-Holland North, Hectorlaan 19, $1702 \mathrm{CL}$ Heerhugowaard, The Netherlands (Europe), Tel: (+3I) 725765 666, Fax: (+3I) 725765 687, E-mail: d.cohen@ggz-nhn.nl
}

mellitus in by far the largest drug-naïve Caucasian population with schizophrenia so far. Second, drug-naïve schizophrenia is, among other aspects, characterized by a 1.27-1.63 times increased prevalence of diabetes mellitus, compared with the general population, which represents the endogenic component of diabetes in schizophrenia. Third, the outcome of the study vindicates the 2009 schizophrenia PORT treatment review that recommends 'antipsychotic medications, other than clozapine and olanzapine, as firstline treatment for persons experiencing their first acute positive symptoms episode' (Buchanan et al, 2010). The position of clozapine-high liability for metabolic side effects, lack of superiority as first-line treatment and proven superior effectiveness in treatment-resistant schizophrenia-does not come as a surprise. The argumentation for olanzapine is twofold, a lack of clinically meaningful differences in efficacy among antipsychotic drugs in meta-analysis, and a significant higher metabolic risk for olanzapine compared with other antipsychotics (RummelKluge et al, 2010). The upshot is a three-phased algorithm for antipsychotic treatment in drug-naïve schizophrenia with FGA or SGA as first-line, olanzapine as second-line and clozapine as third-line treatment.

\section{DISCLOSURE}

Dr Cohen has been a consultant for, received grant/research support and honoraria from, and been on the speakers/ advisory boards of Astra Zeneca, Bristol-Myers Squibb, Eli Lilly, Jansen Cilag. Dr M de Hert has been a consultant for, received grant/research support and honoraria from, and been on the speakers/advisory boards of Astra Zeneca, Bristol-Myers Squibb, Eli Lilly, Janssen-Cilag, Lundbeck JA, Pfizer, and Sanofi Aventis.

\section{REFERENCES}

Buchanan RW, Kreyenbuhl J, Kelly DL, Noel JM, Boggs DL, Fischer BA et al (2010). The 2009 schizophrenia PORT psychopharmacological treatment recommendations and summary statements. Schizophr Bull 36: 71-93. 
Carstensen B, Kristensen JK, Ottosen P, Borch-Johnsen K (2008). The Danish National Diabetes Register: trends in incidence, prevalence and mortality. Diabetologia 51: 2187-2196.

Cohen D (2006). Diabetes mellitus in schizophrenia or schizoaffective disorder: an iatrogenic or endogenic problem? (thesis), Haarlem, Netherlands. ISBN: 90-808509-3-4.

De Hert M, Detraux J, van Winkel R, Correll C (2011). Antipsychotic medications: metabolic, cardiovascular and cardiac risks. Nature Rev Endocr (in press).
Nielsen J, Skadhede S, Correll CU (2010). Antipsychotics associated with the development of type 2 diabetes in antipsychotic-naïve schizophrenia patients. Neuropsychopharmacology 35: 1997-2004.

Rummel-Kluge C, Komossa K, Schwarz S, Hunger H, Schmid F, Asenjo Lobos C et al (2010). Head-to-head comparisons of metabolic side effects of second generation antipsychotics in the treatment of schizophrenia: a systematic review and metaanalysis. Schizophr Res 123: 225-233. 